\title{
Hot injection versus room temperature synthesis of quantum dots: A differential spectroscopic and bioanalyte sensing efficacy evaluation
}

\author{
Irshad AhmedMir ${ }^{1}$, Kishan Das $^{1}$, Kamla Rawat $^{2,3^{*}}$ and H.B. Bohidar ${ }^{1,2 \#}$ \\ ${ }^{1}$ School of Physical Sciences, Jawaharlal Nehru University, New Delhi-110 067, India \\ ${ }^{2}$ Special Centre for Nanosciences, Jawaharlal Nehru University, New Delhi-1 10 067, India \\ ${ }^{3}$ Inter University Accelerator Centre (IUAC), New Delhi 110067, India \\ Email: \# bohi0700@mail.jnu.ac.in, * kamla.jnu@gmail.com
}

Colloidal quantum dots (QDs) have attracted increasing interest to their unique size dependent optical and electronic properties. Some of the most promising applications are imaging and biological sensing, acting as fluorescent probes $[1,2]$. In QD synthesis despite significant progress being achieved, the procedure is still not facile as one would like. A pertinent question arises here. What is the spectroscopic, structural and sensing efficacy (against routine analytes like ascorbic acid, oxalic acid, and citric acid) distinction/difference between the HI- and RTQDs of the comparable size? Hydrogen peroxide plays an important role as a signaling molecule in the regulation of a wide variety of biological processes. Therefore, we specifically targeted $\mathrm{H}_{2} \mathrm{O}_{2}$ as an analyte for detection.

In this work, we have performed an exhaustive comparative study of structural, optical, and sensing properties of CdSe quantum dots (QDs) synthesized by hot-injection (HI), and room temperature (RT) protocols. These were characterized by XRD, TEM, EDX, UV-Vis, and photoluminescence spectroscopy

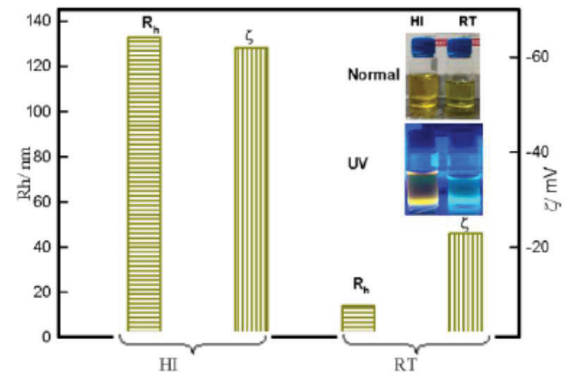

Figure 1: Plot of hydrodynamic radius $\left(\mathrm{R}_{\mathrm{h}}\right)$ and zeta potential $(\zeta$,$) of CdSe quantum dots synthesized by$ the two protocols. Visuals of CdSe QDs synthesized by the two protocols in bright light, and under UV illumination (inset)

However, the optical response profile revealed that HI-QDs had rich spectroscopic signature, but poor sensing attributes (against ascorbic acid, citric acid, oxalic acid, and hydrogen peroxide) compared to RT-QDs. Further, HI-QDs could be synthesized in the size range of 2.5 to $6.3 \mathrm{~nm}$ whereas by RT method only fixed size $(\approx 3.3 \mathrm{~nm})$ nanoparticles could be prepared.

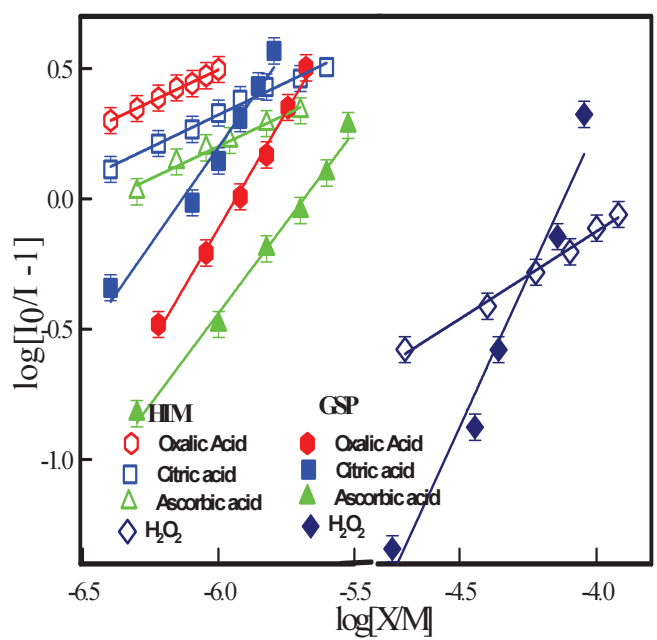

Figure 2: Determination of binding constant, $\mathrm{K}$ and number of binding sites ( $\mathrm{n}$ ) determined from the fluorescence data

Table 1: Calculated binding constant and no. of binding sites

\begin{tabular}{|l|l|l|l|l|}
\hline \multirow{2}{*}{ Analyte } & \multicolumn{2}{|c|}{ HIM } & \multicolumn{2}{l|}{ RT } \\
\cline { 2 - 5 } & $\mathrm{n}$ & $\mathrm{K} \times 10^{3} \mathrm{~L} / \mathrm{M}$ & $\mathrm{n}$ & $\mathrm{K} \times 10^{8} \mathrm{~L} / \mathrm{M}$ \\
\hline $\begin{array}{l}\text { Oxalic } \\
\text { Acid }\end{array}$ & 0.51 & 3.6 & 1.4 & 3.9 \\
\hline Citric Acid & 0.43 & 1.1 & 1.6 & 5.0 \\
\hline $\begin{array}{l}\text { Ascorbic } \\
\text { Acid }\end{array}$ & 0.53 & 2.4 & 1.4 & 8.7 \\
\hline $\begin{array}{l}\text { Hydrogen } \\
\text { Peroxide }\end{array}$ & 0.67 & 3.7 & 2.3 & 3.2 \\
\hline
\end{tabular}

Here $\left(\mathrm{K} / \mathrm{M}^{-1}\right)$ is Binding constant number of binding sites (n) obtained from Figure 2.

\section{References}

1. P. O. Anikeeva, J. E. Halpert, M. G. Bawendi, V. Bulovi'c Nano Letters, 7(8) (2007)2196-2200.

2. I. A. Mir, K. Das, K. Rawat, H. Bohidar, Colloids and Surfaces A: Physicochem. Eng. Aspects 494 (2016) 162169 